\title{
Laser-driven Cs magnetometer arrays for magnetic field measurement and control
}

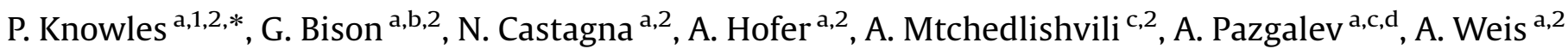 \\ a Department of Physics, University of Fribourg, Switzerland \\ ${ }^{\mathrm{b}}$ Biomagnetisches Zentrum, Klinik für Neurologie, Universitätsklinikum Jena, Germany \\ ${ }^{\mathrm{c}}$ Paul Scherrer Institut, Villigen PSI, Switzerland \\ d Ioffe Physical Technical Institute, St. Petersburg 194021, Russia
}

\begin{abstract}
High precision mapping of weak magnetic fields is of interest for several branches of pure and applied research. This paper presents a resume of the progress made at Fribourg in adapting laboratory style laser-pumped optical Cs magnetometers to multisensor field mapping problems. Progress has been made in sensor head design and manufacture, laser beam splitting and control, and signal treatment electronics. A summary of the technology and its applications are presented.
\end{abstract}

\section{Optical double resonance magnetometers}

Active control of atomic spins by the application of resonant optical and radio frequency (rf) fields has been used since 1950 to measure the coupling of atomic magnetic moments to external magnetic fields, especially when resonance behavior yields information on the fields. For example, discharge-lamp pumped Cs atomic vapors have long been used to measure magnetic field magnitudes in applications as diverse as geological studies and fundamental physics research [1,2]. However, the use of lamps as resonant light sources placed limits on the physical sensor size as well as on the practicality of packing many sensors in close proximity. The arrival of inexpensive laser diodes for heavy alkali spectroscopy opened a new frontier in what was a very mature technology. Laser pumped Cs $\mathrm{M}_{x}$ magnetometers [3] quickly proved to be equal, if not better, in ultimate sensitivity to the existing state-of-the-art lamp-pumped magnetometers [4-6], and quality of and control over the laser light beam permitted a rethinking of the previously highly optimized sensor mechanical design. New directions for research [7-9] and technology applications [10-12], which were previously either impossible or impractical, became possible.

\footnotetext{
* Corresponding author.

E-mail address: paul.knowles@unifr.ch (P. Knowles).

${ }^{1}$ Work funded by the Swiss National Science Foundation, \#200020-111958, and the Velux Foundation.

${ }^{2}$ Working within the PSI nEDM Collaboration.
}

The simplified operating principle of the double resonance orientation magnetometer can be understood with the help of Fig. 1. Circularly polarized resonant light pumps the ground-state magnetic-sublevel population of a Cs atomic vapor to the $6 \mathrm{~S}_{1 / 2}$, $F=4, m_{F}=+3,+4$ dark states-so-called since those specific substates cannot absorb the resonant light and hence do not fluoresce-(Fig. 1, bottom left), leaving the medium transparent. Classically, this is equivalent to the generation of a net macroscopic magnetization $\vec{M}$ (Fig. 1, top left), which precesses at the Larmor frequency, $\omega_{\mathrm{L}}$, in any applied external field $\vec{B}$ (Fig. 1, top right). A small oscillating (or rotating) field $\vec{B}_{\mathrm{rf}}(t)$ destroys the

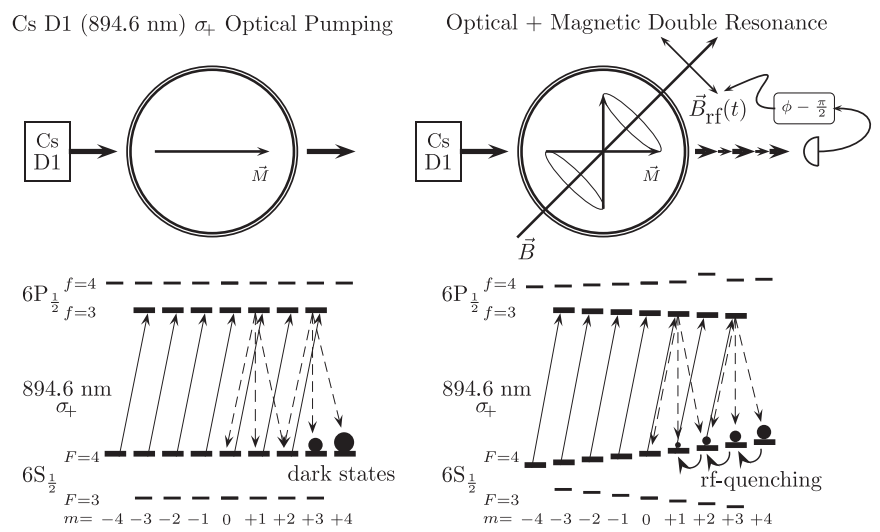

Fig. 1. Double-resonance orientation magnetometer operation. See text for details. 
dark state population (Fig. 1, bottom right), thus the transparency, and so modulates the transmitted light power at the rf frequency, $\omega_{\text {rf }}$, an effect maximized when $\omega_{\text {rf }}=\omega_{\mathrm{L}}$. In its simplest form, a magnetometer is made by extracting the modulation frequency, and, with an appropriate phase shift, using that signal to drive the rf, maintaining the system on resonance and giving the signal

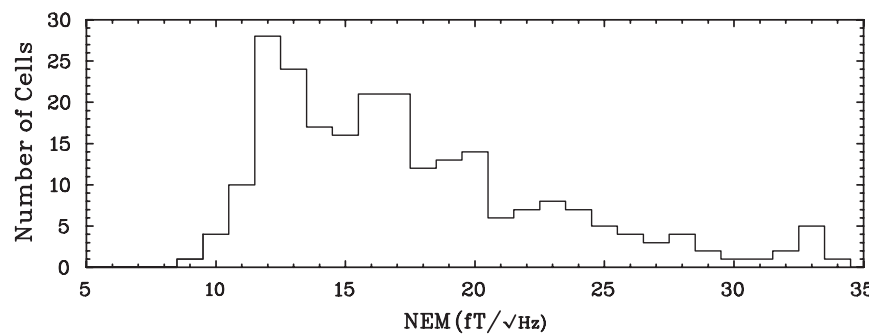

Fig. 2. Histogram of the NEM figure-of-merit for the more than 240 cells manufactured to date. Bins are $1 \mathrm{fT} / \sqrt{\mathrm{Hz}}$ wide. from which $\omega_{\mathrm{L}}$, and via $\omega_{\mathrm{L}}=\gamma|\vec{B}|$ with $\gamma=2 \pi \cdot 3.5 \mathrm{~Hz} / \mathrm{nT}$ (for Cs), $|\vec{B}|$ are determined (Fig. 1, top right). The Dopplerbroadened absorption of the D1 laser is, in general, much broader than the Zeeman splitting, so (ignoring losses to the ground state $F=3$ level) one laser can maintain the $\vec{M}$ polarization.

The precision on the determination of the magnetic field is limited by noise, where the noise equivalent magnetic field (NEM) is

$\delta B=\frac{1}{\gamma} \frac{\Gamma}{S / N}$

with $\Gamma$ the resonance linewidth which depends on both light and rf powers. The signal to noise ratio, $S / N$, is ultimately limited by the photodiode shot noise, although residual laser amplitude noise places the operating point at about $15 \%$ above that limit. Optimized operating conditions, i.e., light and rf powers, maximize $S$, without unduly increasing $\Gamma$ or $N$, and thus minimize $\delta B$. For the magnetometers here, the signal to noise ratio is $S / N \approx 4.2 \times 10^{5} \sqrt{\mathrm{Hz}}$, with $\Gamma=2 \pi \cdot 22 \mathrm{~s}^{-1}$ giving a NEM of $\approx$

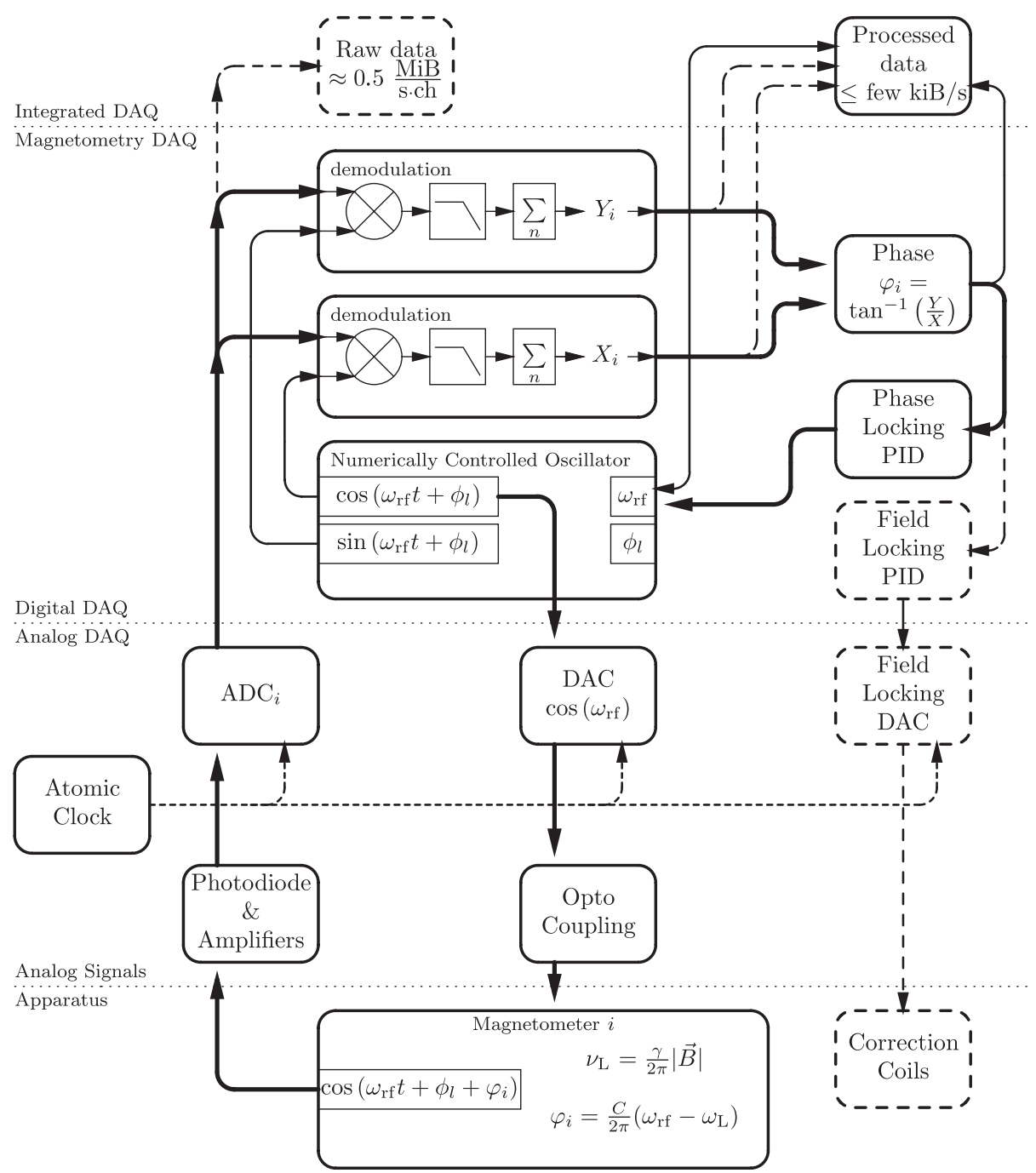

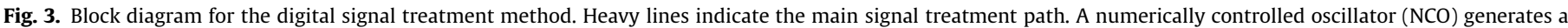

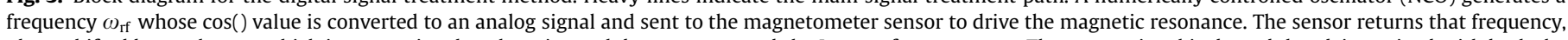

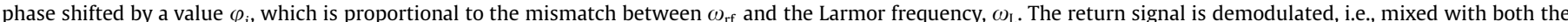

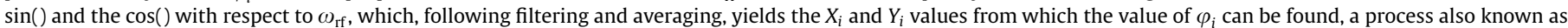

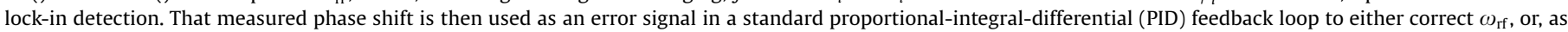

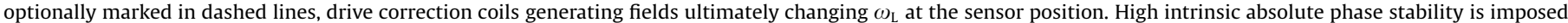
on the whole system by the use of an atomic clock which regulates the conversion ADCs and DACs, as well as the FPGA clock. 
$15 \mathrm{fT} / \sqrt{\mathrm{Hz}}$ where, for uniqueness, the laser power shot noise is used to define $N$. In real conditions where the quadratic Zeeman splitting is unimportant $(|\vec{B}|<25 \mu \mathrm{T}$, for example), sub-pT field changes are readily measurable, a relative sensitivity better than 1 in $10^{7}$.

\section{From research topic to applied technology}

There are three main challenges in adapting a laboratory magnetometer sensor designed for research into a scalable device to be used in an array of sensors useful in applications. They are (1) the production of sufficient antirelaxation coated Cs vapor cells, (2) laser beam splitting and power control, and (3) multichannel signal treatment electronics. The authors have attacked each of those problems in turn, and report here on the progress that has been made.

The Cs vapor referred to in Section 1 and Fig. 1 is at room temperature, and vapor atoms undergo collisions with the walls of the confining cell, and with other Cs atoms, where each collision can lead to a loss of spin polarization (either longitudinal or transverse). Paraffin coatings on the cell walls help prevent loss of coherence. Such evacuated anti-spin-relaxation coated Cs vacuum cells have long been used for magnetic resonance studies [13], yet the manufacture of such cells is an almost artisanal process requiring a skilled glassworking technician using a significant infrastructure of specially designed equipment $[14,15]$. By using laser pumping, the traditional large $(70 \mathrm{~mm})$ diameter cell can be reduced to $25-30 \mathrm{~mm}$ without sensitivity loss, and the smaller size has allowed us to make several cells in parallel, producing up to 10 per week. Cell quality is very uniform, see Fig. 2, with intrinsic magnetic resonance linewidths of $2 \pi \cdot(2-7) \mathrm{Hz}$, the lower value of which is limited by Cs-Cs spin-exchange collisions (for temperature of $21^{\circ} \mathrm{C}$ ). Cell production remains the critical bottleneck in the creation of large magnetometer arrays.

Using discrete components to split a single laser into multiple equal-power beams, each coupled into a fiber, is relatively easy for a few beams, and very difficult for several tens of beams. Our approach uses a hologram which, at present, produces a $5 \times 5$ grid of equal intensity beams from one input. The light spots are focused onto a plate holding a grid of $400 \mu \mathrm{m}$ fibers, resulting in $>40 \%$ power coupling uniform to $\pm 10 \%$ across the grid. The small variations in light power couplings can be matched to the small variations of the cells' optimal pumping powers and so a single hologram input beam power control can bring all magnetometers to their optimum light power operating points. The use of commodity multimode fiber optics permits the sensor heads to be many meters away from the light source, opening the possibility of installing the sensor heads in environments where the large diameter fiber bundles used by lamp pumped magnetometers present operating difficulties (vacuum, high voltage). Long fibers are not without a cost however, acoustic vibrational pickup must be minimized to reduce polarization fluctuations at the fiber output, which increase the noise $N$ and diminish the magnetometric sensitivity, cf. Eq. (1). We have operated magnetometers using $25 \mathrm{~m}$ fibers, and see no a priori reasons, barring the above noise considerations, forbidding source-sensor separations of $10^{3} \mathrm{~m}$ or more.

Signal treatment, which relies on the detection of the phase shift between the input rf frequency and the transmitted intensity modulation, is now made in a fully digital way. With discrete offthe-shelf components, control costs roughly $10 \mathrm{~K} €$ per channel, whereas a single field programmable gate array (FPGA) can host control routines for many (10-100) sensors, reducing the cost to only a few hundred $€$ per sensor. Additionally, treating all signals in the same processor facilitates studying correlations between sensors, and implementing multiorder gradiometers, a powerful field mapping technique.

Fig. 3 shows a block diagram of the signal treatment and explains the steps in the signal treatment. From the electronics viewpoint, the magnetometer is a module into which the frequency $\omega_{\text {rf }}$ is injected (via the rf coils), and which returns a signal (the photocurrent) oscillating at $\omega_{\text {rf }}$ but including an added phase shift $\varphi_{i}$ which is, for frequencies close to the Larmor frequency $\omega_{\mathrm{L}}$, proportional to the difference $\omega_{\mathrm{rf}}-\omega_{\mathrm{L}}$. The figure caption details the extraction of that phase and its use.

\section{Present applications}

As applications of the above technology, the authors have in Fribourg an operating 8 channel second order vertical gradiometer using $50 \times 50 \times 30 \mathrm{~mm}^{3}$ sensors. The devices are spaced with $\Delta x=50 \mathrm{~mm}$ horizontal and $\Delta z=70 \mathrm{~mm}$ vertical separations for cardiomagnetometry, measuring the pT signals of the beating human heart with sub-pT resolution. The only magnetic shielding is a large volume dual-layer $\mathrm{Al}$ conductive shield protecting the sensors from AC field fluctuations. Static coils are used to decrease the ambient earth field and set the value of the offset field needed for optimal sensor operation. The array is installed above the subject positioning bed, used to place the heart in controlled close proximity to the sensor array.

Fig. 4 shows a schematic representation of the eight-sensor array. The signal from the heart, $\vec{B}_{\mathrm{h}}(z)$, falls very sharply with $z$, so the effect of $\vec{B}_{\mathrm{h}}(z)$ is negligible at the reference sensor positions R1 and R2. At R1, the signal is R1 $=B_{0}$. At R2, $B_{0}$ may have changed, so

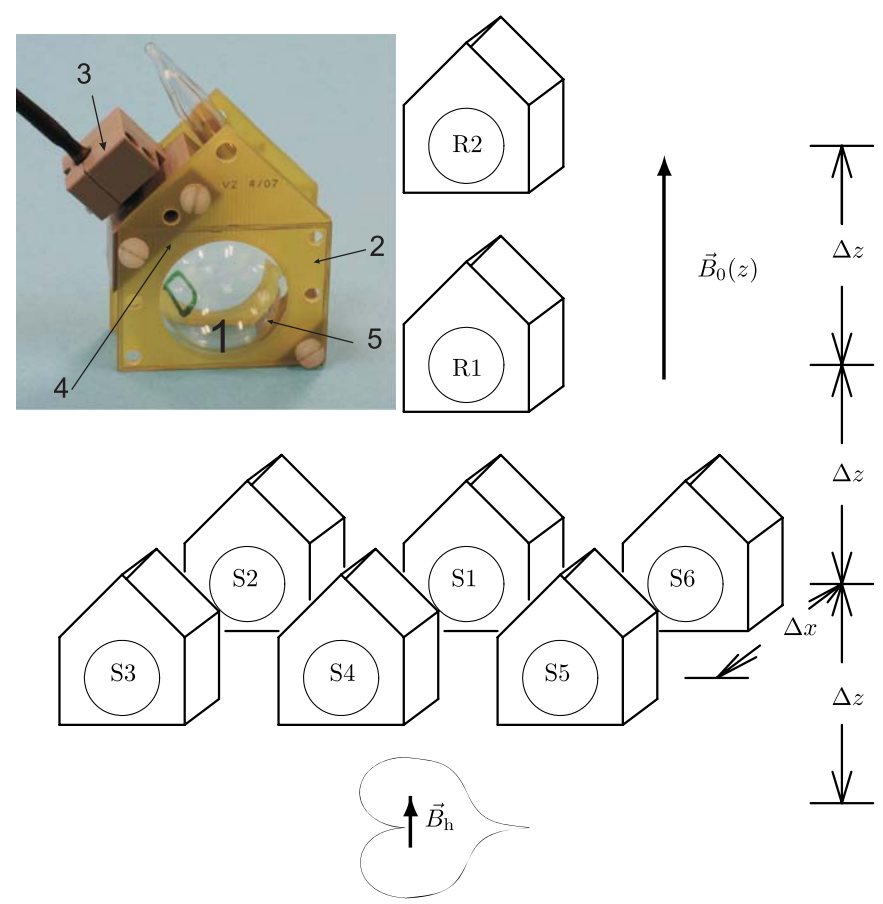

Fig. 4. Inset: photo of the compact magnetometer sensor showing (1) the Cs cell, (2) the printed circuit board supports providing also the $40 \times 30 \mathrm{~mm} \mathrm{rf}$-coils, (3) the optics mounting block for the fiber connector, linear polarizer, and $\lambda / 4$ plate, (4) the point where the laser light enters the cell, and 5) the position of the photodiode. The line drawn schematic is of the 8-sensor, 6 measurement channel (S1-S6), second order gradiometer array configured to measure the magnetic field map of the beating human heart. The sensors are spaced on a grid with separation $\Delta x$, and placed at a height $\Delta z$ above the heart. A static field $\vec{B}_{0}$ fills the whole region of the sensors and source, and provides the offset field in which the sensors operate. Details on the gradiometer operation are given in the text. 


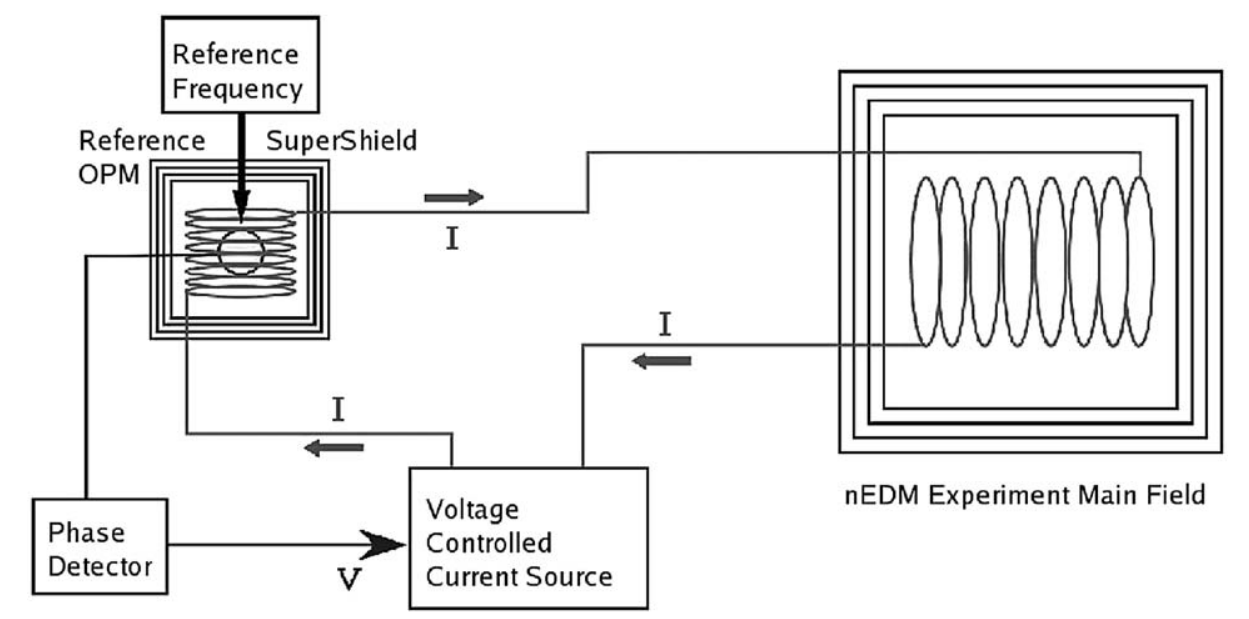

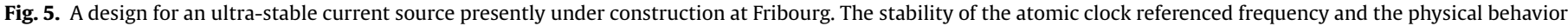
of the well shielded and temperature stabilized solenoid are used to generate a current which can be sent to other experiments.

the signal is represented by a Taylor expansion extrapolated from the R1 position: $\mathrm{R} 2=B_{0}+1 / n !(\Delta z)^{n} \mathrm{~d}^{n} B / \mathrm{d} z^{n}$. The signal in the sensor plane is modelled for any of the six sensor channels S1-S6 by the heart signal and the series expansion $\mathrm{S} x=B_{\mathrm{h}}+B_{0}+(1 / n !)\left((-\Delta z)^{n}\right)\left(\mathrm{d}^{n} B / \mathrm{d} z^{n}\right)$. The combination $\mathrm{S} x+\mathrm{R} 2-2 \mathrm{R} 1$, called the second order gradiometric signal, simplifies to $B_{\mathrm{h}}$ (and higher order even-power derivatives of the field), thus both the field $B_{0}$ and all of its odd-order gradients have been suppressed, which is important when measuring sub-pT effects in $\mu \mathrm{T}$ offset fields.

The FPGA digital control operates the measurement and gradient stabilization. Cardiomagnetic field maps of human volunteers have been made, and with the six-channel system, a medically useful map can be taken in $15 \mathrm{~min}$. A 25 channel device is in preparation for the end of 2008, with 19 dedicated measurement channels, all stabilized by three spatially separated multiorder gradiometers. That array will be able to acquire a full cardiomagnetic field map in under $5 \mathrm{~min}$, a time of interest in a clinical setting. The optical magnetometer maps are competitive with maps acquired by SQUID technology, and hold the promise of being much less expensive to produce and operate.

The same technology will be applied to the PSI nEDM experiment [16], where an array of Cs magnetometers will be deployed inside the multilayer mu-metal shield to monitor and stabilize the magnetic field. Stabilization will be achieved by comparing the Larmor frequency to an atomic clock reference, where the generated error signal will be used via correction coils to modify the $\vec{B}$ magnetic field itself, thus transferring the stability of the atomic clock to the magnetic field of the experiment. Each correction coil within the system will have its own dedicated magnetometer. Additional magnetometers will be placed throughout the volume of the system to monitor the resulting magnetic environment as well as read supplementary field information from free induction decay ${ }^{3} \mathrm{He}$ magnetometers [17].

Fig. 5 shows our proposal for an ultra-stable current source. The above field stabilization method can be inverted, i.e., using firstly an atomic clock disciplined frequency reference and secondly a small highly shielded ultra-stable solenoid to convert a current into a field, and ultimately, via the magnetic sensor, into a frequency. The two frequencies can be locked to each other in a feedback loop, where the current is corrected as needed to keep them equal. Thus, the stability of both the atomic clock and the small magnetometer shielding will be given to the current which can then be used in the primary coil of the main experimental volume.

The future of the multichannel magnetometer approach to field mapping and control holds much promise for a new generation of sensitive experiments, and affordable biomedical instruments.

\section{References}

[1] A.L. Bloom, Appl. Opt. 1 (1962) 61

[2] E.B. Alexandrov, V.A. Bonch-Bruevich, Opt. Eng. 31 (4) (1992) 711.

[3] S. Groeger, G. Bison, J.-L. Schenker, R. Wynands, A. Weis, Eur. Phys. J. D 38 (2) (2006) 239.

[4] E.B. Aleksandrov, M.V. Balabas, A.K. Vershovskii, A.E. Ivanov, N.N. Yakobson, V.L. Velichanskii, N.V. Senkov, Opt. Spectrosc. 78 (2) (1995) 325.

[5] G. Bison, R. Wynands, A. Weis, Appl. Phys. B 76 (3) (2003) 325.

[6] S. Groeger, A.S. Pazgalev, A. Weis, Appl. Phys. B 80 (2005) 645.

[7] A. Weis, G. Bison, A.S. Pazgalev, Phys. Rev. A 74 (3) (2006) 033401.

[8] G. Di Domenico, G. Bison, S. Groeger, P. Knowles, A.S. Pazgalev, M. Rebetez, H. Saudan, A. Weis, Phys. Rev. A 74 (6) (2006) 063415.

[9] G. Di Domenico, H. Saudan, G. Bison, P. Knowles, A. Weis, Phys. Rev. A 76 (2) (2007) 023407.

[10] G. Bison, R. Wynands, A. Weis, Opt. Expr. 11 (2003) 904.

[11] S. Groeger, G. Bison, A. Weis, J. Res. Natl. Inst. Stand. Technol. 110 (3) (2005) 179.

[12] G. Bison, R. Wynands, A. Weis, J. Opt. Soc. Am. B 22 (2005) 77.

[13] M.A. Bouchiat, J. Brossel, Phys. Rev. 147 (1) (1966) 41.

[14] G. Singh, P. Dilavore, C.O. Alley, Rev. Sci. Instrum. 43 (1972) 1388.

[15] M.T. Graf, D.F. Kimball, S.M. Rochester, K. Kerner, C. Wong, D. Budker, E.B. Alexandrov, M.V. Balabas, V.V. Yashchuk, Phys. Rev. A 72 (2) (2005) 023401

[16] M. Burghoff, et al., these proceedings.

[17] Y. Borisov, W. Heil, M. Leduc, V. Lobashev, E.W. Otten, Y. Sobolev, Nucl. Instr. and Meth. A 440 (2000) 483. 